\title{
The MESSIER surveyor: unveiling the ultra-low surface brightness universe
}

\author{
David Valls-Gabaud ${ }^{1,2}$ and the MESSIER collaboration \\ ${ }^{1}$ CNRS, Observatoire de Paris, France \\ ${ }^{2}$ Institute of Astronomy, Cambridge, UK
}

\begin{abstract}
The MESSIER surveyor is a small mission designed at exploring the very low surface brightness universe. The satellite will drift-scan the entire sky in 6 filters covering the 200$1000 \mathrm{~nm}$ range, reaching unprecedented surface brightness levels of 34 and $37 \mathrm{mag} \operatorname{arcsec}^{-2}$ in the optical and UV, respectively. These levels are required to achieve the two main science goals of the mission: to critically test the $\Lambda$ CDM paradigm of structure formation through (1) the detection and characterisation of ultra-faint dwarf galaxies, which are predicted to be extremely abundant around normal galaxies, but which remain elusive; and (2) tracing the cosmic web, which feeds dark matter and baryons into galactic haloes, and which may contain the reservoir of missing baryons at low redshifts. A large number of science cases, ranging from stellar mass loss episodes to intracluster light through fluctuations in the cosmological UV-optical background radiation are free by-products of the full-sky maps produced.
\end{abstract}

Keywords. Cosmology, dwarf galaxies, stellar populations, cosmic web, intergalactic medium, diffuse radiation, space instrumentation

\section{Introduction: driving science cases}

The current $\Lambda \mathrm{CDM}$ cosmological paradigm has been extraordinarily successful at accounting for observations of the cosmological background and the formation and evolution of galaxies. The bottom-up scenario of galaxy formation driven by the growth of dark matter haloes makes two generic predictions that hitherto have not been observed: the existence of a large-scale network of filaments, extending over hundreds of Mpc, and the existence of a large number of satellite galaxies. The discrepancy between the observed number of satellites in the Milky Way and in M31 and the theoretical predictions is very serious and may point to processes preventing the infall of baryons in small haloes through feedback mechanisms or reionisation effects. It may also provide a clue on the nature of dark matter: warm or self-interacting dark matter would erase small-scale fluctuations, alleviating the crisis. In addition, these dwarf galaxies appear to be the most dark matter-dominated objects in the universe, making them ideal laboratories to explore the nature of dark matter. Its large-scale distribution in a cosmic web of filaments populated by diffuse baryons also remains elusive.

Remarkably, in spite of the widely different scales (in the linear and highly non-linear regimes), these two predictions share one common property: their surface brightness are orders of magnitude below the night sky foreground. Indeed, all the satellites of the Galaxy discovered thus far through resolved star counts have extremely low surface brightness, well below 28 mag $\operatorname{arcsec}^{-2}$. If fainter and/or located at larger distances, this population would remain undetectable. Progress in these two fields can only be achieved by measuring ultra-low surface brightness levels, which are unreachable from the ground. 
Table 1. Top-level scientific requirements

\begin{tabular}{lcll}
\hline \multicolumn{1}{c}{ Quantity } & Symbol & \multicolumn{1}{c}{ Value } & \multicolumn{1}{c}{ Comment } \\
\hline Field of view & FOV & $>2^{\circ} \times 2^{\circ}$ & lifetime of satellite \\
Focal ratio & $f / D$ & $<3$ & $100 \times$ better than HST in SB \\
Central obscuration & $\epsilon$ & none & minimal PSF wings \\
Pixel size & $\theta_{p i x}$ & 1 arcsec & matches ground \\
Wavelength coverage & $\Delta \lambda$ & $150-1000 \mathrm{~nm}$ & stellar populations and cosmic web \\
Flat-field accuracy & $\epsilon_{f f}$ & $<0.0025 \%$ & implies TDI/drift-scan \\
Diameter & $D$ & $50 \mathrm{~cm}$ & set by the size of the platform \\
Survey solid angle & $\Omega$ & $4 \pi$ (full sky) & unique mission \\
\hline
\end{tabular}

\section{The MESSIER surveyor}

The reason is that current instrumentation is not optimised for the discovery of extended, low surface brightness objects (but see Trujillo \& Fliri 2016). Telescopes have been designed to detect point-like sources such as stars or distant galaxies, where the flux received scales as $F_{\text {point }} \sim(D / 2)^{2} t_{\exp } 10^{-0.4 m}$. In contrast, the observed surface brightness scales as $\mathrm{SB}_{\text {extended }} \sim(f / D)^{-2} t_{\text {exp }} s_{\text {pix }}^{2} N_{\text {pix }} 10^{-0.4 \mu}$, and hence fast optics with a minimal $f / D$ ratio are required. A second complication comes from the extended and chromatic PSFs whose wings produce fake haloes and bias the photometry of extended objects (e.g. Sandin 2014). The huge and deserved success of the Dragonfly camera (Abraham \& van Dokkum 2014) arises from the mitigation of both factors. Ground-based observations are however limited by the sky background, its variability in time and direction, straylight contamination, extended PSF wings due to the scattering of atmospheric aerosols and molecules, and the accuracy of flat fielding on large scales. For a proper control on the systematics, observations from space are compulsory. Given the scientific requirements (Table 1), an optimal solution with an off-axis TMA with free-form mirrors has been designed (Hugot et al. 2014), with no lenses (which produce Cherenkov radiation). The flat focal plane is divided into 12 independent controllers of EMCCDs and CMOS, each optimised for the filters required (Fig. 1). With this design (Fig. 2), and a $700 \mathrm{~km} \mathrm{SSO}$ orbit, the mission reaches the goal of measuring SB levels of $34 \mathrm{mag} \operatorname{arcsec}^{-2}$ in the 4 optical filters designed to characterise unresolved stellar populations. At $200 \mathrm{~nm}$, minimising cosmological dimming, an optimal combination of a narrow- and a broad-band filter allows MESSIER to reach $37 \mathrm{mag} \operatorname{arcsec}^{-2}$, the expected level of emission in Ly $\alpha$ from the cosmic web at $z \sim 0.65$ (Bertone \& Schaye 2012; Silva et al. 2016) as well as from extended galactic haloes.

\section{Other science cases}

Since MESSIER opens the last niche that remains to be explored in parameter space, its discovery potential is huge. In addition to the two science drivers, and as free by-products, the following additional science cases have been identified: the actual luminosity function of galaxies, the properties of intracluster light, the prevalence of warm $\mathrm{H}_{2}$ (detected in Lyman-Werner bands) in galaxies at $z \sim 0.25$, the BAO scale at $z \sim 0.65$ with over $310^{6}$ galaxies detected in $\mathrm{Ly} \alpha$, the UV/optical cosmological background and its fluctuations, the cosmological distance scale with SB fluctuations, simultaneous UVoptical light curves of SN, GRB and TDEs, mass loss episodes in giant stars, extended debris discs, the polarisation by dust grains, and the reference space-based photometric catalogue of point sources. The wide wavelength coverage and angular resolution ensures an optimal removal of the foregrounds (zodiacal light and dust cirri). Further details on the proposed mission can be found at http://messier-orbiter.obspm.fr 


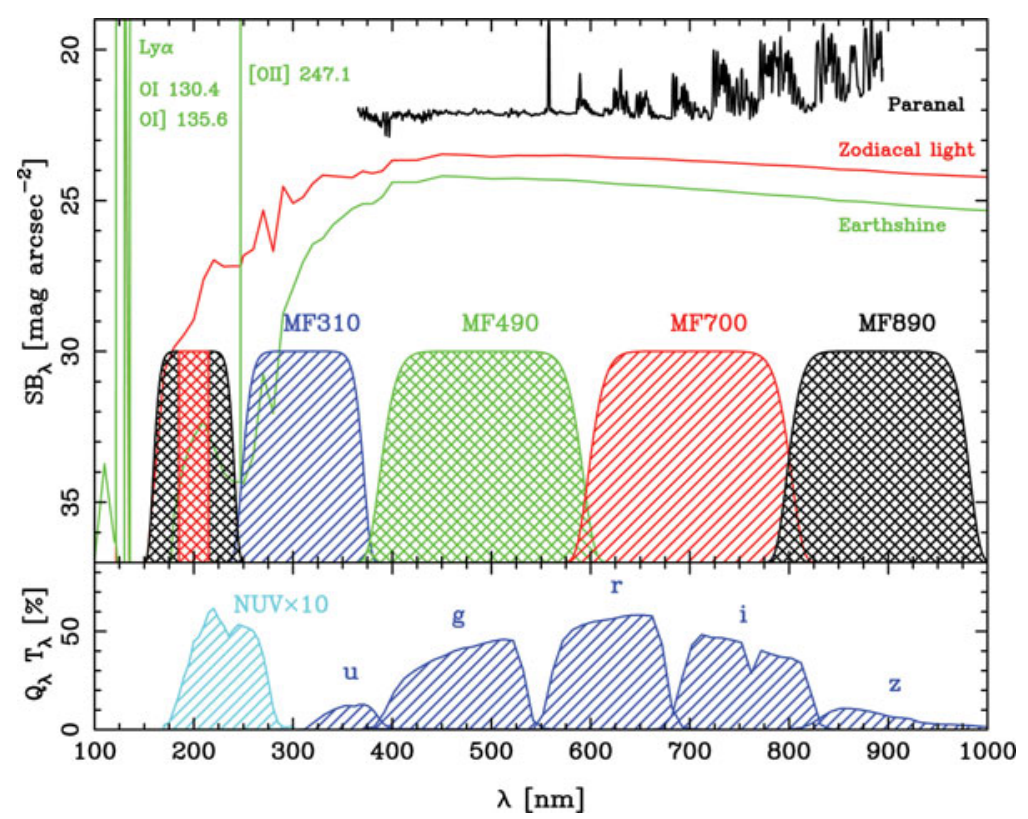

Figure 1. Top: The surface brightness of the sky at Paranal compared with the emission of the zodiacal light and the earthshine as measured by HST. Not only the strongly variable sky emission is no longer an issue in space, but in addition the gain is over 4 mag $\operatorname{arcsec}^{-2}$ at red wavelengths, and at $200 \mathrm{~nm}$ these two foregrounds become negligible while keeping $Q_{\lambda} T_{\lambda} \sim$ $85 \%$. The proposed set of filters for MESSIER, optimised for characterising unresolved stellar populations and measuring the cosmic web is also shown. Bottom: The overall throughput of the SDSS, and GALEX's NUV (multiplied 10×). MESSIER is more efficient in SB than any other telescope ever by over two orders of magnitude across the full 200-1000 nm range.
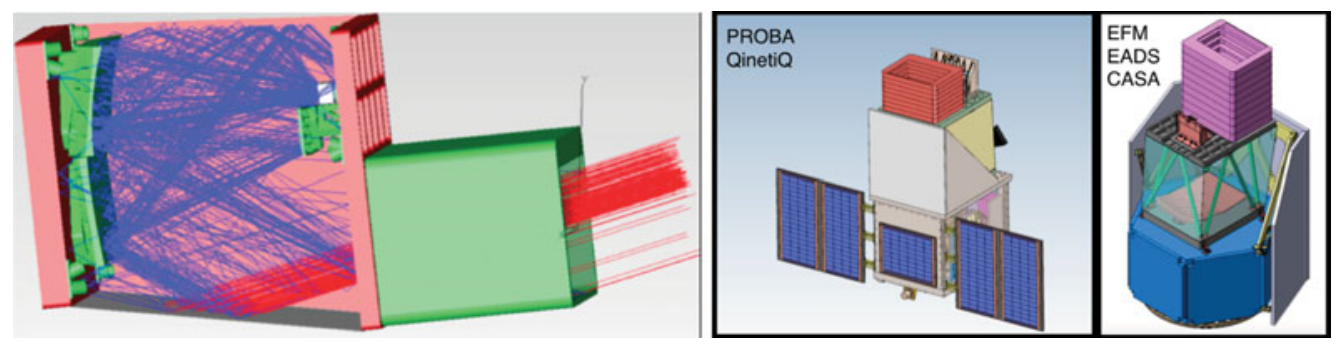

Figure 2. Left: The proposed payload is an off-axis TMA with free-form mirrors, providing a $2^{\circ} \times 4^{\circ}$ FOV at $f / 2.0$. The flat focal plane is covered by EMCCDs for the UV channels, and CMOS in the optical domain. With no moving parts, passive cooling, and a scanning strategy, the observing efficiency is maximised. The straylight mitigation is provided by a pop-up baffle. Right: Designs provided by the industrial contractors (QinetiQ, left, and EADS CASA, right), using the PROBA-V and EFM platforms, respectively, ensuring that all the requirements on attitude control, telemetry, power and downlink rates are met.

\section{References}

Abrahan, R. \& van Dokkum, P. 2014, PASP, 126, 55

Bertone, S. \& Schaye, J. 2012, MNRAS, 419, 780

Hugot, E., Wang, X., Valls-Gabaud, D. et al. 2014, Proc. of the SPIE, Vol. 9143, id. 91434X

Sandin, C. 2014, A\& A, 567, A97

Silva, M. B., Kooistra, R., \& Zaroubi, S. 2016, MNRAS, in press (arXiv:1603.06952)

Trujillo, I. \& Fliri, J. 2016, ApJ, 823, 123 